\title{
Nutzungsherausgabe und Verjährung beim Verkauf gestohlener Sachen: Nichterfüllung oder Rechtsmangel?
}

Von Dr. Sonja Meier, Hamburg

\section{Einleitung}

Es ist eine bekannteErfahrung, dass man bei der Lösung scheinbar einfacher Fälle nach neuem Leistungsstörungsrecht immer wieder auf Probleme stößt - neuartige oder auch altvertraute. Hier geht es um den Anspruch auf Nutzungsherausgabe beim Verkauf abhanden gekommener Sachen: $V$ erwirbt gutgläubig einen dem $\mathrm{E}$ gestohlenen LKW, den er an $\mathrm{K}$ verkauft und gegen Barzahlung übergibt. Nach sechs Monaten vindiziert $\mathrm{E}$ (der wegen $\S 935$ Abs. 1 Satz $1^{1}$ Eigentümer geblieben ist) den LKW von K. Er ist zur Veräußerung an V oder zur Genehmigung der Veräußerung an $\mathrm{K}$ nur zu einem unverhältnismäßsig hohen Preis bereit.

Geht man von der Annahme aus, dass wegen der fehlenden Übereignung an $\mathrm{K}$ ein Fall der Nichterfüllung vorliegt, kann V gegen K's Erfüllungsanspruch aus $\S 433 \mathrm{Abs}$. 1 Satz 1 die Einrede aus $\S 275$ Abs. 2 erheben, womit sowohl K's Erfüllungsanspruch untergeht als auch, nach $\S 326$ Abs. 1 Satz 1, V's Kaufpreisanspruch aus $\S 433$ Abs. 2. K kann den gezahlten Kaufpreis zurückverlangen, §§ 346 Abs. 1, 326 Abs.4. Angenommen, V habe wegen perfekter Fälschung der Fahrzeugpapiere nicht erkennen können, dass er einen gestohlenen LKW erwarb, scheitert auch ein Schadensersatzanspruch des $K$ wegen anfänglichen Leistungshindernisses aus $\S 311$ a Abs. 2 Satz 1, da eine nicht zu vertretende Unkenntnis i.S. d. § 311 a Abs. 2 Satz 2 vorliegt. Die frühere Garantiehaftung bei anfänglichem Unvermögen sollte nach Vorstellung des Reformgesetzgebers durch diese Vorschrift abgeschafft werden ${ }^{2}$. V verlangt nun von $\mathrm{K}$ den Wert der sechsmonatigen Nutzung des LKW. Die Frage ist, ob das neue Leistungsstörungsrecht dafür noch eineAnspruchsgrundlage bereithält.

\section{Keine Anspruchsgrundlage für die Nutzungs- herausgabe?}

Der an sich passende $\$ 346$ Abs. 1 i.V. m. Abs. 2 Nr. 1 setzt einen Rücktritt voraus, der nicht erfolgt ist. Zwar verweist auch § 326 Abs. 4 auf die§§ 346-348, doch nur, soweit die nicht geschuldete Gegenleistung bewirkt ist, also für die Rückforderung des gezahlten Kaufpreises durch K. Der Ausdruck »das Geleistete« könnteallerdings dem Wortlaut nach von der nicht geschuldeten Gegenleistung abgekoppelt und als »das beiderseits Geleistete« gelesen werden. Diese Lesart würde aber dazu führen, dass nach $\S 326$ Abs. 4 diegegenseitigeRückforderung des Geleisteten dann stattfindet, wenn der Käufer schon gezahlt hat. Steht der Kaufpreis dagegen noch aus, könnte der Verkäufer weder Rückgabe noch Nutzungsersatz verlangen. Der Anspruch des Verkäufers kann aber nicht davon abhängen, ob der Käufer schon gezahlt hat. Der Ausdruck »das Geleistete« in $\S 326$ Abs. 4 bezieht sich auf die nicht geschuldete Gegenleistung allein. An einen Rückforderungsanspruch des Schuldnersder unmöglichen Leistung hat der
Gesetzgeber in § 326 nicht gedacht. Liegt also eine Regelungslücke vor?

$M$ an wende nicht ein, $V$ solle auch gar keinen Wertersatzanspruch für die Nutzungen haben, weil diese dem $V$ als Nichtberechtigtem auch nie zugestanden hätten, $\mathrm{K}$ den LKW daher auf Kosten des E, nicht des V, genutzt habe. Der Berechtigte E kann nämlich von $K$ wegen $\S 993$ Abs. 1 nichts verlangen. Angemessen ist daher, dass $\mathrm{K}$ die Nutzungen mit seinem Vertragspartner $\mathrm{V}$ abrechnet, gegenüber dem er auch den Anspruch auf Rückzahlung des Kaufpreises hat. Nur auf diese Weise bleibt dem $\mathrm{E}$ zumindest die $\mathrm{M}$ öglichkeit, über $\mathrm{V}$ und seine Vormänner den Wert der Nutzung schließlich doch noch zu erhalten ${ }^{3}$.

Nach altem Recht konnte $\mathrm{V}$ den Wert der Nutzungen nach $\S 327$ Satz 2 BGB a. F. i.V.m. § 818 Abs. 1 (nach anderer Ansicht aus $\S 327$ Satz 1, 347 Satz 2, 987 Abs. 1 BGB a. F.) verlangen. Grund dafür war die Garantiehaftung für anfängliches Unvermögen, die dem K die Rechte aus § 326 Abs. 1 BGB a. F. (oder, nach h.L., § 325 Abs. 1 BGB a.F. analog) eröffnete ${ }^{4}$. Wollte $K$ danach den Kaufpreis zurückverlangen, musste er zurücktreten. $M$ it dem Rücktritt aber war auch die Herausgabe gezogener Nutzungen verbunden.

Im neuen Recht dagegen geht der Gegenleistungsanspruch des $\checkmark$ nach $\S 326$ Abs. 1 automatisch unter, ohne dass es eines Rücktritts bedarf, der einen Anspruch auf Nutzungsersatz eröffnen würde. Grundsätzlich hat der Gesetzgeber in Rückabwicklungskonstellationen stets dafür gesorgt, dass alles, was seitens des pflichtverletzenden Schuldners geleistet wurde, zurückgegeben werden muss: nicht nur im Falle eines Rücktritts (wozu K hier theoretisch nach $\S 326$ Abs. 5 berechtigt wäre, was inm aber wegen § 326 Abs. 1 keinen Vorteil brächte), sondern auch wenn $\mathrm{K}$ Schadensersatz statt der Leistung verlangt (etwa wenn $\mathrm{V}$ eine Garantie abgegeben hätte), § 311 a Abs. 2 Satz 3 i.V.m. § 281 Abs. 5. Selbst der Fall der Nachlieferung bei mangelhaften Leistungen wurde nicht übersehen ( $\S 439$ Abs. 4, 635 Abs. 4). Nur

1 Paragraphen ohne Angaben beziehen sich auf das BGB in der aktuell geltenden Fassung.

2 Vgl. Canaris, JZ 2001 S. 499 (506), und DB 2001 S. 1815 (1818f.) Wer die Einstandspflicht des Schuldners für sein anfängliches Leistungsvermögen auch im neuen Recht beibehalten will (indem er mit dem »Inhalt des Schuldverhältnisses« oder mit einer Garantie i.S. von § 276 Abs. 1 Satz 1 arbeitet), mögefür den vorliegenden Zweck annehmen, $\mathrm{K}$ habekein Interesse an einem Schadensersatzanspruch, sondern begnüge sich mit der Rückforderung des Kaufpreises.

3 Im Falle einer geschlossenen Veräußerungskette zwischen dem Dieb und $\mathrm{K}$ würden die von $\mathrm{K}$ gezogenen Nutzungen an den jeweiligen Vorgänger zurückerstattet, bis sie der Dieb erhält, von dem sie der Eigentümer aus $\S 687$ Abs. 2 und aus $\S \S 987,989$ herausverlangen kann. Ist ein Glied der Kette nicht mehr auffindbar, verbleiben die Nutzungen bei demjenigen Käufer, der seinen Verkäufer nicht mehr ausfindig machen kann. Dies ist nicht ungerecht, da er dann auch den gezahlten Kaufpreis nicht zurückverlangen kann.

4 Siehe stellvertretend $\mathrm{H}$ u ber, Leistungsstörungen I, 1999, § 23 I m. w. N. 
beim automatischen Wegfall der Gegenleistung nach § $326 \mathrm{Abs} .1$ fehlt eine Anspruchsgrundlage auf Rückgabe einer schon erbrachten Leistung. Der Reformgesetzgeber ging davon aus, dass esim Fall des§ $326 \mathrm{Abs}$. 1 per definitionem keineschon erbrachte Leistung geben kann, die zurückgegeben werden könnte. Die Leistung ist vollständig unmöglich und wird daher nicht erbracht. Auch bei einer Teilunmöglichkeit ist keine besondere Anspruchsgrundlage für die Rückgabe der schon erbrachten Teilleistung erforderlich: Entweder entfällt auch nur der entsprechende Teil der Gegenleistung, so dass der Gläubiger die empfangene Teilleistung behält. Oder der Gläubiger hat an der Teilleistung kein Interesse und will daher seine gesamte Gegenleistung zurück; in diesem Fall muss er nach $\S 326$ Abs. 5 zurücktreten, womit zugleich der Anspruch des Schuldners auf Rückgabe der Teilleistung eröffnet wird.

Nur dieÜ bergabe der Kaufsache ohneÜ bereignung will nicht in dieses Schema passen. Sie gilt traditionell als Fall der Nichterfüllung/Unmöglichkeit, obwohl der Verkäufer dem Käufer zumindest zeitweise den Besitz ermöglicht. Demnach würde beim Verkauf einer abhanden gekommenen Sache nach neuem Recht keinerlei Rückgabepflicht des Käufers bestehen, obwohl er nach $\S 326$ Abs. 1 und 4 den Kaufpreis zurückerhält. Tatsächlich könntesich K, da§ 440 Abs. 2 bis 4 BGB a. F. weggefallen ist, auch ohneVindikation durch $\mathrm{E}$ auf den Standpunkt stellen, er fordere den Kaufpreis aus $\S \S 326$ Abs. 1 und 4, 346 Abs. 1 zurück und behaltemangels Anspruchsgrundlage des V den LKW. Das kann nicht richtig sein.

Falls es sich hier um eine Gesetzeslücke handelt, beruht sie nicht unbedingt auf einem Fehler des neuen Rechts. Dieselbe Lücke findet sich nämlich auch im alten Leistungsstörungsrecht, nur etwas versteckter. Einen automatischen Wegfall des Gegenleistungsanspruchs gab es dort nach $\S 323$ Abs. 1 BGB a. F. im Fall nachträglicher unverschuldeter Unmöglichkeit. Angenommen, $V$ verkauft und übergibt dem $\mathrm{K}$ ein Grundstück, das nach sechs M onaten, noch vor Auflassung und Eintragung, vom Staat enteignet wird: Dann läge nach altem Recht wohl ein Fall der nachträglichen nicht zu vertretenden Unmöglichkeit vor ${ }^{5}$. K könnte einen schon gezahlten Kaufpreis nach § 323 Abs. 1 und 3 BGB a. F. i.V.m. § 812 Abs. 1 zurückverlangen. Will V Wertersatz für die sechsmonatige Nutzung, fehlt auch hier eine Anspruchsgrundlage. Auch bei $\S 323$ Abs. 1 BGB a. F. ging der Gesetzgeber davon aus, dass sich das Problem der Rückforderung (und damit verbunden des Nutzungsersatzes) einer Leistung bei automatischem Wegfall der Gegenleistungspflicht nicht stellt. Auch hier sollte eine Teilunmöglichkeit nur zur M inderung der Gegenleistung führen. Ein Recht des Käufers, trotz Teilleistung wegen Inter essewegfalls die gesamte Gegenleistung zurückzuerhalten, sah das alte Recht nicht vor. Wer, wie Otto, trotzdem ein solches Recht annahm, brauchte eine Anspruchsgrundlage für den Verkäufer zur Rückforderung der erbrachten Teilleistung, die vom Gesetz nicht vorgesehen war. Otto wendete daher $\S 323$ Abs. 3 BGB a. F. analog an 6 .

EineAnalogiekämeauch für dasvorliegendeProblem in Frage: Die Anspruchsgrundlage des $\S 326$ Abs. 4, die ihrem Wortlaut nach nur für die Rückforderung der Gegenleistung gilt, könnte analog auf die Rückforderung durch den Schuldner angewendet werden, dessen Leistung als unmöglich gilt und der trotzdem etwas geleistet hat.

\section{Lieferung ohne Eigentumsverschaffung: Nichterfüllung oder Rechtsmangel?}

Das ganze Problem und die vermeintliche Gesetzeslücke würde im neuen Recht aber verschwinden, wenn man die Lieferung ohne Eigentumsverschaffung nicht als Fall der Nichterfüllung betrachtet, sondern dem Regime für Rechtsmängel unterstellt. Der Käufer hätte dann nicht mehr den Erfüllungsanspruch aus
$\S 433$ Abs. 1 Satz 1, sondern den Nacherfüllungsanspruch aus $\S 439$ Abs. 1, der durch die Einrede des Verkäufers nach § 275 Abs. 2 untergeht. Der Kaufpreisanspruch des Verkäufers entfällt dann wegen $\S 326$ Abs. 1 Satz 2 nicht automatisch. Vielmehr muss der Käufer, will er den gezahlten Kaufpreis zurück, entweder nach $\S 326$ Abs. 5 zurücktreten und/oder, falls möglich, aus $\S 311$ a Abs. 2 Schadensersatz verlangen. In beiden Fällen entsteht ein Anspruch des Verkäufers auf Rückgabe seiner Leistung (beim Schadensersatz nach § 311 a Abs. 2 Satz 3, 281 Abs.5) und damit auch auf Nutzungsersatz aus $\S 346$ Abs. 1.

Kann man aber von einem Rechtsmangel sprechen, wenn der Verkäufer Besitz ohneEigentum verschafft? Im alten Recht findet sich zu dieser Frage verhältnismäßig wenig. Der BGH hatte sich lediglich mit der Frage zu beschäftigen, ob es sich bei der bloßen Besitzverschaffung ohne Eigentum um eine Teilleistung handelt, was er entschieden verneinte: Sei nur die Lieferung, nicht die Eigentumsübertragung möglich, scheitere die Erfüllung des Kaufvertrags im Ganzen; insofern liege vollständige Unmöglichkeit vor ${ }^{7}$. In den Kaufrechtskommentaren zum alten Recht ging die ganz herrschende Lehre davon aus, dass Rechtsmängel im Sinnedes§ 434BGB a. F. nur beschränktedinglicheRechteD ritter seien: Die Lieferung völlig fremder, insbesondere gestohlener Sachen verletze ja schon die Pflicht zur Eigentumsverschaffung

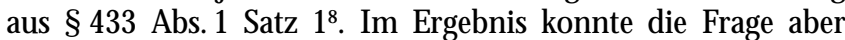
dahingestellt bleiben, weil die Rechtsfolgen einer Verletzung des $\S 433$ Abs. 1 Satz 1 und des § 434 BGB a. F. dieselben waren ${ }^{9}$ : Stets blieb dem Käufer sein Erfüllungsanspruch; und über $\S 440$ Abs. 1 BGB a. F. konnteer die Rechtsbehelfedesal Igemeinen Leistungsstörungsrechts geltend machen. Rechtsmangel und Nichterfüllung waren keine Gegensätze.

Es bildete daher keinen Widerspruch, die Unmöglichkeit der Eigentumsverschaffung einerseits als Verletzung der Pflicht aus $\S 433$ Abs. 1 Satz 1 und nicht aus § 434 BGB a.F. anzusehen, andererseits, soweit es um die Anwendung anderer Vorschriften ging, sie als Rechtsmangel zu bezeichnen. Bei § 439 BGB a. F., der die Rechte des Käufers bei Kenntnis des $M$ angels ausschloss, war es umstritten, ob seine Anwendung bei fehlender Eigentumsverschaffung sinnvoll (als Auslegungsregel) ${ }^{10}$ oder unpassend (weil die Garantiehaftung bei Beschaffungsschulden unterlaufend ${ }^{11}$ ist. Soweit es um die Beweislastregel des§ 442 BGB a. F. ging, war man sich dagegen verhältnismäßig einig, dass die Vorschrift auch bei fehlender Eigentumsverschaffung anwendbar sein sollte ${ }^{12}$. DieFrage, was ein »Rechtsmangel « war, hing von der Auslegung der betreffenden Gesetzesbestimmung ab. So lehnte etwa der

5 Ein vergleichbarer Sachverhalt lag dem BGH -Urteil vom 7. 2. 1969 - V ZR 112/65, NJW 1969 S. 837, zugrunde. Der Anspruch auf Nutzungsersatz wurde aber nicht erörtert.

60 t t 0 , in: Staudinger, BGB, N eubearbeitung 2001, § 323 Rdn. 52.

7 BGH-Úrteil vom 7. 2. 1969, a. a. O. (Fn. 5); BGH-Urteil vom 30. 10. 1998 V ZR 3677-997, NJW-RR 1999 S. 346 (347)

8 So ausdrücklich H u ber , in: Soergel, BGB, 12. Aufl. 1991, § 434 Rdn. 32; P u t z 0, in: Palandt, BGB, 61. Aufl. 2002, § 434 BGB a. F. Rdn. 4; W a I t er, Kaufrecht, 1987, § 4 I 1; K n öpf I e, NJW 1991 S. $889 \mathrm{f}$. In der Kommentierung von K ö h l er , in: Staudinger, BGB, 13. Aufl. 1995, § 434 Rdn. 6, und bei R ei n i cke/T i ed t ke, Kaufrecht, 6. Aufl. 1997, werden nur beschränktedinglicheRechteerwähnt. Anders (Lieferung gestohlener Sachen fällt unter $\S 434$ BGB a. F.) nurV o I I k o m mer , in:Jauernig, BGB, 9. Aufl. 1999, § 434 Rdn. 4.

9 So Grunewald, in: Erman, BGB, 10.Aufl. 2000, § 434 Rdn. 1; W est er ma n n, in: M ünchKomm., BGB, 3. Aufl. 1995, § 434 Rdn. 13.

10 So Kö h l er, a.a. O. (Fn. 8), § 439 Rdn. 4; G r u n ewal d, a.a. O. (Fn. 9), $\S 439$ Rdn. 1; L a r en z, Schuldrecht II 1, 13. Aufl. 1986, § 40 II b.

11 So H u ber , a.a. O. (Fn. 8), § 439 Rdn. 4, 10 ff. m. w. N. zum Streitstand; West er man $n$, a.a.0. (Fn. 9), § 439 Rdn. 2.

12 Vgl. Köhler, a.a.O. (Fn. 8), § 442 Rdn. 2; W est er mann, a.a.O. (Fn. 9), § 442 Rdn. 2; H uber, a.a. O. (Fn. 8), § 439 Rdn. 13. Nach K nö pf I e, NJW 1991 S. 889 f., kann die Unmöglichkeit der Eigentumsverschaffung dagegen grundsätzlich nie Rechtsmangel sein. 
BGH es ab, die Privilegierung des Schenkers bei seiner Haftung für Rechtsmängel nach $\S 523$ BGB a. F. auf die $\mathrm{H}$ aftung für das anfängliche Unvermögen zur Eigentumsverschaffung anzuwenden ${ }^{13}$. Die Literatur ist dem entgegengetreten ${ }^{14}$, wohl zu Recht: DieH aftungsmilderung soll geradeauch denjenigen erfassen, der eine vermeintlich eigeneSache verschenkt, dieihm aber in Wahrheit nicht gehört. DieUnmöglichkeit der Ü bereignung ist Rechtsmangel i.S.d. § 523, selbst wenn es gar nicht zur Besitzverschaffung kommt, wenn der Gläubiger also überhaupt nichts erhalten hat.

Die Frage, ob eine Lieferung ohne Eigentumsverschaffung Nichterfüllung oder Rechtsmangel ist, stellte sich im alten Recht also nicht, weil Nichterfüllung und Rechtsmangel sich nicht ausschlossen. Im neuen Leistungsstörungsrecht ist dies anders. Der Reformgesetzgeber hat zwar einerseits die Haftung für Sachmängel und Rechtsmängel vereinheitlicht, andererseitsaber auch den bisherigen Gleichlauf der Haftung für Nichterfüllung und der Haftung für Rechtsmängel abgeschafft. Sobald man einen Leistungsstörungstatbestand als Rechtsmangel i. S. d. § 435 einordnet, ergeben sich unterschiedliche Rechtsfolgen im Vergleich zur Nichterfüllungshaftung:

(1) Bei einem Rechtsmangel ist der automatische Wegfall des Gegenleistungsanspruchs nach § 326 Abs. 1 Satz 2 ausgeschlossen, wodurch der Käufer zum Rücktritt und damit zur Herausgabe des Empfangenen bzw. zur Nutzungsherausgabe gezwungen wird. (2) Nur bei einem Rechtsmangel besteht das Recht des Käufers zur Minderung nach §§ 437 Nr. 2, 441. (3) Im Falle eines Rechtsmangels kann der Käufer bei Kenntnis oder grob fahrlässiger Unkenntnis seine Rechte verlieren, $\S$ 442. (4) Schließlich bestehen Unterschiede bei der Verjährung:

Sieht man die fehlende Übereignung als Nichterfüllung an, hat der Käufer bei Unmöglichkeit der Eigentumsverschaffung einen »gewöhnlichen « Schadensersatzanspruch aus §311 a Abs. 2, der in der regelmäßigen Frist von drei Jahren (bei beweglichen Sachen) verjährt, $\S 195$. Vindiziert der wahre Eigentümer zwölf Jahre nach Abschluss des Kaufvertrags, so bildet diese Frist kein Problem, weil sie nach § 199 Abs. 1 erst beginnt, nachdem der Käufer Kenntnis von den anspruchsbegründenden Umständen, also vom Unvermögen des Verkäufers, erlangt hat, was gewöhnlich erst bei der Vindikation stattfindet. Probleme bereitet aber die zehnjährige H öchstfrist des § 199 Abs. 3 N r. 1, die schon mit Entstehung des Anspruchs zu laufen beginnt. Der Schadensersatzanspruch aus $\S 311$ a Abs. 2 entsteht schon mit Abschluss des Kaufvertrags, da es sich um anfängliches Unvermögen handelt und die Vorschriften des $\S 440$ Abs. 2 bis 4 BGB a. F. im neuen Recht weggefallen sind. Während also der Eigentümer 30 Jahre lang vindizieren kann, § 197 Abs. 1 Nr. 1, kann sich der Käufer, nimmt man Nichterfüllung an, nur bis zu zehn Jahre nach Abschluss des Kaufvertrags an den Verkäufer halten ${ }^{15}$.

Nimmt man dagegen einen Rechtsmangel an, ist der Anspruch des Käufers auf Verschaffung des fehlenden Eigentumsein Nacherfüllungsanspruch i.S.d. §§ 437 Nr. 1, 439. Sowohl dieser Anspruch als auch der Schadensersatzanspruch aus $\S 311$ a Abs. 2 verjähren nach § 438 Abs. $1 \mathrm{~N} \mathrm{r}$. 1 a in 30 Jahren ab Ablieferung der Sache. Diese 30 jährige Verjährungsfrist geht auf die Vorschläge von Ernst/Gsell und Mansel zurück: Wenn Dritte, die ein dingliches Recht an der Kaufsache haben, nach § 197 Abs. 1 N r. 130 Jahre lang H erausgabeansprüche gegenüber dem Käufer geltend machen können, argumentierten sie, müssedem Käufer auch die Möglichkeit gewährt werden, ebenso lang Regress gegenüber dem Verkäufer zu nehmen ${ }^{16}$. Dabei bezog $M$ ansel in seine Argumentation ausdrücklich den Fall ein, dass der Käufer gar kein Eigentum erhalten hatte. Tatsächlich ist der durch § 438 erreichte Gleichlauf der Verjährungsfristen für den dinglichen Herausgabeanspruch des Dritten einerseits und für den Regress des
Käufers andererseits ein starkes Argument, die Lieferung ohne Eigentumsverschaffung in die Rechtsmängelhaftung des $\S 435$ einzuordnen.

Demzufolge geht auch die Literatur zum neuen Schuldrecht mehrheitlich, aber ohne vertiefte Diskussion, davon aus, dass es sich beim Verkauf gestohlener Sachen um einen Rechtsmangel i.S.d. $\S \S 435,437$ handelt ${ }^{17}$. Häufig findet sich lediglich der Hinweis, die Verjährungsregelung des $\S 438$ Abs. 1 Nr. 1 a sei auch beim Verkauf gestohlener Sachen anwendbar ${ }^{18}$. Dass damit die Lieferung ohne Eigentumsverschaffung in Änderung des bisherigen Rechts aus der Nichterfüllungshaftung herausgenommen ist, wird dabei vielleicht nicht immer ganz klar $^{19}$. Die Anwendung der Rechtsmängelhaftung würde bedeuten, dass der Käufer nun erstmalswegen fehlender Eigentumsverschaffung den Kaufpreis mindern kann ${ }^{20}$. Sie würde ferner dazu führen, dass der Käufer, der das anfängliche Unvermögen des Verkäufers zur Eigentumsverschaffung grob fahrlässig nicht erkannt hat, seine Rechte gegenüber dem Verkäufer wegen § 442 ganz verlieren kann. Vor allem aber würde beim Verkauf und der Lieferung gestohlener Sachen der Erfüllungsanspruch desKäufers aus $\S 433$ Abs. 1 Satz 1 ganz untergehen und dem Nacherfüllungsanspruch aus $\S 437$ Platz machen, obwohl der Verkäufer seiner Pflicht zur Eigentumsverschaffung aus § 433 Abs. 1 Satz 1 überhaupt nicht nachgekommen ist.

\section{Zusammenfassung}

Im Ergebniserscheint dieEinordnung der Lieferung ohneEigentumsverschaffungin dieRechtsmängelhaftung sowohl wegen der

13 BGH -Urteil vom 23. 3. 2000 - X ZR 177/97, ZIP 2000 S. 1059

$14 \mathrm{H}$ u ber , ZIP 2000 S. 1372; L ö w i s c h , JZ 2001 S. 355. Das Problem stellt sich auch im neuen Recht: Haftet der eine vermeintlich eigene Sache Verschenkende für die Unmöglichkeit der Übereignung gemäß $§ 523$ Abs. 1 nur bei Arglist oder schon bei Fahrlässigkeit i. S.d. § 311 a Abs. 2? 15 Noch unbefriedigender ist das Ergebnis, wenn man annimmt, der Eigentümer sei zur Veräußerung zu einem angemessenen Preis bereit, so dass eine Einrede aus $\S 275$ Abs. 2 nicht erhoben werden kann. Der Käufer hätte in diesem Fall den Erfüllungsanspruch aus $\S 433$ Abs. 1 Satz 1 auf Verschaffung desEigentums. Hier wärewohl nicht nur dieZehnjahresfrist des $\S 199$ Abs. 3 Nr. 1, sondern schon die Dreijahresfrist des $\S 195$ ein Problem. Denn die den Anspruch begründenden Umstände, die der Gläubiger kennen muss, damit die Verjährung nach $\S 199$ Abs. 1 beginnt, sind wohl allein der wirksame Kaufvertrag. Im Ergebnis wären dann K's Ansprüche gegen $V$ bei nicht möglicher Eigentumsverschaffung nach zehn Jahren, bei noch möglicher Eigentumsverschaffung dagegen schon nach drei Jahren verjährt: Auch dies kann nicht richtig sein.

16 Vgl. die Begründung zum Regierungsentwurf, BT-Drs. 14/6040 S. 227; Ernst / G sel I, ZIP 2000 S. 1812; M a n sel, in: Ernst/Zimmermann (H rsg.), Zivilrechtswissenschaft und Schuldrechtsreform, 2001, S. 353.

17 E h ma n n / Su t sc h et, M odernisiertes Schuldrecht, 2002, S. 237 Fn. 2, 243, 246; Lor en z/R i eh m, Lehrbuch zum neuen Schuldrecht, 2002, Rdn. 570 f.; D a u n e r - L i e b u. a., Fällezum neuen Schuldrecht, 2002, Fall 156, S. 312 f.; B er g er , in: Jauernig, BGB, 10. Aufl. 2003, § 435 Rdn. 5; W est er mann, NJW 2002 S. 241 (246); Eiden mül I er, NJW 2002 S. 1625 (1626); C o est er - W a I t jen, JURA 2002 S. 534 (539). Anders offenbar 0 I zen / W a n k, Die Schuldrechtsreform, 2002, vgl. Rdn. 78 und 365 .

18 So M a n sel , in: Dauner-Lieb u.a. (H rsg.), Das neue Schuldrecht, 2002, $\S 1$ Rdn. 139; W it t , in: Schwab/Witt, Einführung in das neue Schuldrecht, 2002, S. 37 Fn. 65; B u c k , in: Westermann (H rsg.), Das Schuldrecht 2002, 2002, S. 159; H a a s, BB 2001S. 1313(1318); H ei n r i c h s, BB 2001 S. 1417 (1420); Bal I , ZGS2002 S. 49 (53).

19 So hält P u t zo beim Verkauf gestohlener Sachen § 438 für anwendbar; zugleich soll aber die Nichtverschaffung des Eigentums nicht unter $\S 435$, sondern unter $\S 433$ Abs. 1 Satz 1 fallen: Palandt, BGB, 62. Aufl. 2003, $\S 435$ Rdn. 8; § 438 Rdn. 6. Auch Sa en g er erwähnt die Lieferung ohne Eigentumsverschaffung zwar bei $\S 438$, nicht aber bei $\S 435: \mathrm{H}$ andK, BGB, 2. Aufl. 2002, § 435 Rdn. 3; § 438 Rdn. 5. Ähnlich H a a s, Das neue Schuldrecht, 2002, Kap. 5, Rdn. 134 und 312.

$20 \mathrm{Zu}$ berücksichtigen ist aber, dass das $M$ inderungsrecht für Rechtsmängel ohnehin neu ist und dass ein Minderungsrecht bei der Lieferung gestohlener Sachen nicht notwendig zu unerwünschten Ergebnissen führen muss. 
Verjährungsregelung des $\S 438$ als auch im Hinblick auf den Nutzungsersatzanspruch des Verkäufers als sinnvoll. In Kauf nehmen muss man dabei allerdings den Systembruch, dass die Nichterfüllung der Pflicht zur Eigentumsverschaffung aus $\S 433$
Abs. 1 Satz 1 zugleich als Rechtsmangel i. S. d. § 433 Abs. 1 Satz 2 gilt. Dies aber ist nur eine der vielen Unstimmigkeiten, die bei Anwendung des reformierten Leistungsstörungsrechts in Kauf genommen werden müssen. 